\title{
Electrocardiogram in Chagas disease: can anything be learned from an old tool?
}

\author{
Alexandre C. Pereira ${ }^{[1]}$ \\ [1]. Laboratório de Genética e Cardiologia Molecular, Instituto do Coração, Faculdade de Medicina, Universidade de São Paulo, São Paulo, SP, Brasil.
}

\begin{abstract}
Chagas disease (CD) has been a persistent public health issue for most Latin American countries. The need for perennial vector control programs and the establishment and maintenance of resource-rich infrastructures for the care of individuals with chronic forms of the disease have been challenges to countries with often unstable economic and political environments. Additionally, increasing immigration of the Latin American populations to the United States and Europe has posed significant challenges to the healthcare systems of developed countries that must adapt to the problems imposed by this disease ${ }^{1}$.

In this issue Brito et al. ${ }^{2}$ reviewed the role of electrocardiogram, a proven and inexpensive tool in the management of chronic Chagas disease ${ }^{2}$. Used since the discovery of this disease, the electrocardiogram has established itself as an indispensable tool in the diagnosis and management of patients with Chagas cardiomyopathy.

In their review, the authors nicely describe the evidence regarding the robust associations between electrocardiogram findings and a number of important hallmarks in CD progression, from the diagnosis of the chronic form of the cardiomyopathy, to the uses of electrocardiographic features to predict heart failure, ventricular arrhythmias, stroke, and death. Of major
\end{abstract}

importance is the proposition made by the authors for the use of composite scores that leverage electrocardiographic variables to derive more accurate predictors of disease development and incidence of late stage events. If successful, this may greatly expand the use of the electrocardiogram in poor, remote areas that are also currently experiencing the highest increases in incidence for the disease.

This timely review re-emphasizes the benefits of using this inexpensive and proven technology and reveals areas where small investments in research can bring cost-beneficial results to societies that experience the greatest burden of the disease.

Conflict of interest

The author declares that there is no conflict of interest.

\section{REFERENCES}

1. Manne-Goehler J, Umeh CA, Montgomery SP, Wirtz VJ. Estimating the burden of Chagas disease in the United States. PLoS Negl Trop Dis. 2016:10(11):e0005033.

2. Brito BOF, Ribeiro ALP. Electrocardiogram in Chagas disease. Rev Soc Bras Med Trop. 2018;51(5):570-577. 